\title{
Research Square \\ High expression of RRM2 promotes the \\ pathogenesis of malignant ovarian endometriosis.
}

\section{Binkai Yang}

Tianjin Medical University

\section{Yuanjing Hu ( $\nabla$ hyj_tdj@sina.com )}

Tianjin Central Hospital of Obstetrics and Gynecology https://orcid.org/0000-0002-4631-4115

\section{Tian Wang}

Tianjin Central Hospital of Obstetrics and Gynecology

\section{$\mathrm{Na} \mathrm{Li}$}

Tianjin Central Hospital of Obstetrics and Gynecology

\section{Wenwen Zhang}

Tianjin Central Hospital of Obstetrics and Gynecology

\section{Research Article}

Keywords: EC, OE, EAOC, Hub gene, RRM2

Posted Date: April 2nd, 2021

DOI: https://doi.org/10.21203/rs.3.rs-342433/v1

License: (c) (1) This work is licensed under a Creative Commons Attribution 4.0 International License. Read Full License 


\section{Abstract}

Objective: Our objective was to investigate the upregulated expression of ribonucleotide reductase M2 (RRM2) in the ectopic endometrium (EC) of ovarian endometriosis (OE) patients that may indicate malignant transformation. RRM2 may be used as a marker of $\mathrm{OE}$, which contribute to the research of the mechanism of the malignant transformation of $\mathrm{OE}$.

Methods: The gene expression profiles of ovarian cancer and OE were downloaded from Gene Expression Omnibus (GEO), and a common hub gene, RRM2, was identified. The expression of RRM2 was low in OE and high in ovarian cancer. A total of 44 patients with endometriosis-associated ovarian cancers (EAOC) and 44 with $\mathrm{OE}$ were enrolled in this study. Immunohistochemistry (IHC) and real-time quantitative polymerase chain reaction (RT-qPCR) were used to detect the expression of RRM2, while the relationship between RRM2 and Ki-67 was analyzed by IHC co-localization.

Results: There was no significant difference in the expression of RRM2 in the eutopic endometrium (EU), EC, and cancer tissues of EAOC patients. Compared with OE patients, the mRNA and protein expression levels of RRM2 were higher in the EC of EAOC patients ( $\mathrm{p} \otimes 0.01)$. Moreover, the high expression of RRM2 was consistent with the expression of $\mathrm{Ki}-67$ in EC of EAOC patients.

Conclusions: The upregulated expression of RRM2 in the EC of OE patients may indicate malignant transformation. RRM2 may be used as a marker of $\mathrm{OE}$, which allows the investigation of the mechanism of the malignant transformation of $\mathrm{OE}$.

\section{Introduction}

Around $10 \%$ of women of childbearing age have endometriosis (EMS), which is defined as the appearance of a functional endometrium outside the uterine cavity, resulting in difficulty in menstruation, sexual intercourse, pelvic pain, and infertility [1, 2]. EMS is considered to be a benign disease; however, many cases have shown features common to malignant tumors, such as peripheral tissue invasion, atypical histology, distant metastasis, and recurrence after treatment [3]. Previous studies also found that around $1 \%$ of EMS cases could result in malignant changes, of which the most common is ovarian lesions [4-6]. Endometriosis-associated ovarian cancers (EAOCs) including clear cell ovarian carcinoma $(\mathrm{CCOC})$ and endometrioid ovarian carcinoma (EOC) are a relatively rare subtype of ovarian cancer $[7,8]$. There is sufficient evidence showing that women with histologically confirmed EMS have an increased risk of developing CCOC and EOC [9-11]. The relationship between EMS and ovarian cancer can be divided into the transition from EMS to invasive ovarian cancer or the coexistence of ovarian cancer and EMS without transition $[12,13]$. Sampson first proposed and described the mechanism and process of the malignant transformation of EMS in 1925; however, the exact mechanism is still unclear [14].

NCBI-GEO OE and ovarian cancer datasets were used to screen for differentially expressed genes (DEGs) with low expression in OE and high expression in ovarian cancer. These DEGs were used to construct the protein-protein interaction (PPI) network, and the top five hub genes were examined: TOP2A, MKI67, 
RRM2, CCNB2, and CDK1. In addition, literature related to OE and ovarian cancer hub genes was reviewed, which revealed that RRM2 and CCNB2 are also the hub genes of the two diseases [15-17]. The difference in the expression of RRM2 was greater than that of CCNB2; thus, RRM2 was selected as the target gene in this study.

Ribonucleotide reductase (RNR) catalyzes the conversion of ribonucleoside 5'-diphosphate to 2'deoxyribonucleoside 5'-triphosphate (dNTP), which is an essential DNA component for DNA replication and repair [18]. RRM2 is a regulatory subunit of RNR and is essential for the biogenesis of dNTP in the S phase of the cell cycle in which DNA replication occurs [19]. Tumor cells are characterized by uncontrolled proliferation, and DNA synthesis is necessary for this process. RRM2 is considered as a potential target for the development of cancer therapy due to its active role in tumorigenesis[20, 21]. Hyperphysiological RRM2 inhibits oncogene-induced senescence (OIS) and promotes genomic instability by allowing errorprone replication due to the imbalance of the dNTP pool, which ultimately promotes tumorigenesis [22]. The overexpression of RRM2 has been reported to be related to the resistance of cancer cells to various chemotherapeutic drugs $[23,24]$. In addition, in some cancer cells, high levels of RRM2 expression are associated with cell invasion, tumor metastasis, angiogenesis, and poor prognosis [25-29]. Studies have shown that the expression of RRM2 in epithelial ovarian cancer is positively correlated with Ki67, a marker of cell proliferation [30]. RRM2 interacts with a various of oncogenes to promote tumor progression, suggesting that RRM2 may be involved in the malignant progression of tumors [31]. However, it is unclear whether there is a significant difference in the expression of RRM2 protein between ovarian cancer and OE and if a difference in expression is related to the malignant transformation of OE. Data analysis indicated that the expression of RRM2 was low in EMS and high in ovarian cancer. Therefore, we hypothesized that the expression of RRM2 in the malignantly transformed EC may be upregulated. The upregulation of RRM2 may play an important role in the process of the malignant transformation of $\mathrm{OE}$.

In this study, we obtained the cancer tissue, EC, and EU samples from patients with EAOC, which was caused by OE. In addition, EC samples were obtained from OE patients. The expression of RRM2 mRNA and protein in these tissues was assessed. The objectives of the study were analyzing the promotion of the malignant transformation of OE caused by the high expression of RRM2 and predicting this process.

\section{Materials And Methods}

\subsection{Data sources}

The NCBI-GEO database is a free and public database containing gene profiles. Two microarray datasets of ovarian cancer (GSE26712, GSE6008) and two microarray datasets of OE(GSE7305,GSE25628) were obtained from the GEO database (https://www.ncbi.nlm.nih.gov/gds/). The inclusion criteria for the above gene expression profiles were set as follows: (1) the tissue samples should consist of from human pathological tissues and normal tissues; (2) the number of samples in each dataset should be more than 
8; (3) all selected datasets should be provided by Affymetrix platforms to reduce the 'platform effect' due to different probe designs by different companies.

\subsection{Data preprocessing and analysis}

The OE and ovarian cancer datasets were processed as follows: (1) Identification of DEGs: R package limma was applied to filter the DEGs in the datasets; (2) Screening of DEGs: DEGs with low expression in OE and high expression in EAOC were screened; (3) PPI network construction $\square T$ The PPI network of the identified DEGs was constructed using an online tool, the Search Tool for the Retrieval of Interacting Genes/Proteins (STRING; https://string-db.org/), with an interaction score of > 0.4; (4) Hub gene selection and analysis: The PPI network was visualized using Cytoscape software (version 3.8.2), and the top 5 hub nodes in the network were identified.

\subsection{Ethics statement}

The study plan was approved by the Human Ethics Committee of Tianjin Central Obstetrics and Gynecology Hospital. All subjects gave informed consent to participate in this study.

\subsection{Patients}

Although EOC and $\mathrm{CCOC}$ are collectively referred to as EAOC, there is no requirement that both cancers should be caused by OE. Therefore, the criterion for screening patients in this study was EAOC patients whose cancerous lesions have been confirmed by pathology to be the malignant transformation of $\mathrm{OE}$. A total of 44 patients with EAOC diagnosed as the malignant transformation of OE from September 2010 to March 2020 were selected, including 24 cases of CCOC and 20 cases of EOC. A total of 44 patients with $\mathrm{OE}$ in the same period were also recruited.

\subsection{Collection of samples and immunohistochemical staining}

Formalin-fixed, paraffin-embedded (FFPE) sections were obtained from the cancer tissue, EC, and EU samples collected from 44 EAOC patients with OE malignancy (24 patients with CCOC and 20 patients with EOC). The FFPE sections of EC samples from 44 OE patients in the same period were also obtained.

The slides were dewaxed in xylene and rehydrated in water by fractionated ethanol before dyeing. Antigen retrieval was performed with 1 mM EDTA buffer solution ( $\mathrm{pH} \mathrm{9.0)} \mathrm{for} \mathrm{in} \mathrm{all} \mathrm{sections,} \mathrm{and} \mathrm{the} \mathrm{endogenous}$ peroxisomal enzymes were inactivated by $3 \% \mathrm{H} 2 \mathrm{O} 2$. After blocking for $30 \mathrm{~min}$, the cells were incubated overnight with the antibodies RRM2 (11661-1-AP, 1:150 dilution; ProteinTech) and Ki-67 (ZM-0166, 1:150 dilution; ZSGB-BIO) at $4^{\circ} \mathrm{C}$. After washing, the slides were stained with secondary antibodies for 30 min at room temperature. Diaminobenzidine and hematoxylin were used as the chromogenic substrate and for nuclear counterstaining, respectively. For the negative control, each antibody was replaced with phosphate buffer solution. Five random visual fields $(\times 400)$ were observed under an optical microscope. The results of immunohistochemical staining were evaluated independently by two pathologists who were blinded to the clinical data. In the tissue core containing tumor cells and stromal cells, the intensity 
of the tumor cells was scored according to morphological criteria. The expression level of RRM2 was determined based on the percentage of positive cells. Without knowing the background of the samples, two experienced gynecological pathologists evaluated the samples using histopathology and immunostaining scores.

\subsection{Quantification of staining intensity and percentage}

The expression of RRM2 was classified according to a grading system. Scores that corresponded to the percentages of stained cells were defined as follows: 0 for no positively stained cells; 1 for $₫ 25 \%$ positively stained cells; 2 for $25 \%-50 \%$ positively stained cells; 3 for $\otimes 50 \%$ positively stained cells. Moreover, in terms of the intensity of the stain, the following scores were designated: 0 for no staining; 1 for weak staining; 2 for moderate staining; 3 for strong staining. The immunostaining score for RRM2 expression was represented as the sum of the percentage score and the intensity score. The expression of RRM2 was finally defined as follows: "low expression (-)" for a score of $\leq 2$; "medium expression (+)" for a score of 3 or 4 ; and "high expression (++)" for a score of 6 or 9 . Two independent observers who were blinded to the sample background evaluated the cell staining.

\subsection{RNA extraction and RT-qPCR}

The FFPE sections of EC samples from 15 patients with EAOC diagnosed pathologically as the malignant transformation of OE from 2015 to 2020 were obtained. There were 10 cases of CCOC and 5 cases of EOC. The FFPE sections of EC samples from 15 patients with OE in the same period were also obtained.

The miRNeasy FFPE Kit (Qiagen) was used to isolate total RNA from FFPE tissue sections. Purified RNA $(2 \mu \mathrm{g})$ was used for reverse transcription with the miScript II RT Kit (Qiagen). RT-qPCR was carried out with the CFX96 Touch Real-time PCR Detection System (Bio-Rad) using the Sso Advanced SSO SYBR Green Supermix (Bio-Rad).

\subsection{Statistical analysis}

SPSS 18.0 software was used for statistical analysis. Data are presented as the mean \pm SD, and Student's t-test was used to calculate the $P$ value. Two-sided $P$ values of $<0.05$ were considered statistically significant.

\section{Results}

\subsection{Identification and screening of DEGs}

Four expression profiles (GSE6008, GSE26712, GSE7305, and GSE7307) were obtained from the GEO database. GSE6008 consisted of 99 ovarian tumor cases and 4 controls. GSE26712 contained 185 ovarian tumor cases and 10 controls. GSE7305 included 10 OE cases and 10 controls. GSE7307 contained 18 cases and 22 controls. The DEGs in the four datasets (4079 DEGs in GSE6008, 4115 DEGs in GSE26712, 5100 DEGs in GSE7305, and 881 DEGs in GSE7307) were extracted after gene expression 
profile data processing and standardization with $\mathrm{P}<0.05$ and $|\log \mathrm{FC}|>2$. The up-regulated and downregulated genes belonging to each gene set are shown in Fig. 1(a-d). DEGs with low expression in OE and high expression in ovarian cancer were screened. The overlapping DEGs among these four datasets included 86 genes as shown in the Venn diagram (Fig. 1e).

\subsection{Selection and analysis of hub genes by PPI network construction}

In order to find the hub genes involved in OE processed to ovarian cancer. The STRING online database was used to construct the PPI network of the hub genes, and Cytoscape software was used to draw the interaction network of the hub genes and their related genes. The PPI network of the hub genes consisted of 86 nodes and 319 edges, and the PPI enrichment P value was lower than $1.0 \mathrm{e}-16$ (Fig. 2a). The top 5

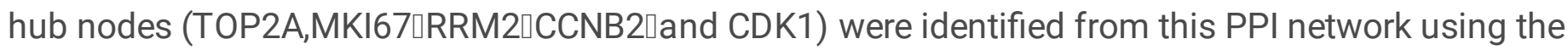
Degree method in plugin Cytohubba (Fig. 2b). We further reviewed the literature on the hub genes of ovarian cancer and OE. RRM2 and CCNB2 were identified as hub genes in ovarian cancer and OE. According to DEG analysis, the difference in the expression of RRM2 was greater than that of CCNB2; thus, we selected RRM2 as the target gene in this study (Fig. 2c).

\subsection{Expression of RRM2 in EAOC patients at different pathological stages}

We performed IHC for RRM2 in the EU, EC, and cancer tissue samples of EAOC patients (CCOC and EOC) (Fig. 3). There was a significant expression of RRM2 protein in cancerous tissue of CCOC and EOC (Fig. 3). The expression of RRM2 was not affected by the type of cancer (Fig. 3). There was no significant low expression of RRM2 protein between the two groups of EC samples compared with the corresponding EU and cancer tissue samples, and the expression of RRM2 was significant in both groups (Fig. 3).

\subsection{Comparison of RRM2 expression in EC of EAOC and OE patients}

The results of IHC clearly showed that the expression of RRM2 in EC of EAOC was much higher than that of OE(Fig. 4a). We scored the staining of RRM2 in the EAOC and OE of EC samples (Fig. 4b). The results of immunohistochemical scoring showed that there was a significant difference in the expression of RRM2 in the EC of EAOC and OE patients (Fig. 4b). Around 26\% of patients with EAOC had a high expression of RRM2 protein in the EC; however, no high expression of RRM2 protein was observed among patients with OE (Table 1). Around $69 \%$ of patients with OE had a low expression of RRM2 protein in the EC, whereas only $5 \%$ of patients with EAOC had a low expression of RRM2 (Table 1). The mRNA level of RRM2 extracted from the FFPE sections of EC samples was also significantly higher in EAOC cases than in $\mathrm{OE}$ cases $(\mathrm{P}<0.01)$ (Fig. 4c). 
Table 1

Protein expression of RRM2 in EC of EAOC and OE

\begin{tabular}{|c|c|c|c|}
\hline Parameters & ectopic lesions & & $P$ value \\
\hline & $\mathrm{EAOC}(\mathrm{n}=44)$ & $\mathrm{OE}(\mathrm{n}=44)$ & \\
\hline Age $^{\mathrm{a}}$ at operation (years) & $49(28-65)$ & $49.5(25-67)$ & 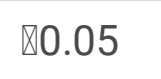 \\
\hline RRM2 expression & $\llbracket 0.01$ & & \\
\hline Low* & $5(11 \%)$ & $27(61 \%)$ & \\
\hline medium & $13(30 \%)$ & $17(39 \%)$ & \\
\hline High & $26(59 \%)$ & 0 & \\
\hline \multicolumn{4}{|c|}{$\begin{array}{l}\text { * Low }=\text { low RRM2 expression (+); Medium = medium RRM2 expression }(++) ; \text { High = high RRM2 } \\
\text { expression }(+++) .\end{array}$} \\
\hline \multicolumn{4}{|l|}{ a Median } \\
\hline
\end{tabular}

\subsection{IHC co-localization of RRM2 and $\mathrm{KI}-67$ in EC of EAOC patients}

$\mathrm{Ki}-67$ is an indicator of cell proliferation. We intended to observe the proliferation of tissue in the EC of EAOC expressed by RRM2. The results of the immunohistochemical co-localization of Ki-67 and RRM2 showed the co-expression of RRM2 and Ki-67 (Fig. 5).

\section{Discussion}

The molecular mechanism underlying the malignant transformation of $\mathrm{OE}$ remains controversial, and the exact mechanism of carcinogenesis has not been clarified. The process of the malignant transformation of $\mathrm{OE}$ into ovarian cancer is complex, and the mechanism is different from that of the transformation of normal ovarian epithelial tissue into ovarian cancer tissue. A difficulty in the study of the mechanism of malignant transformation of $\mathrm{OE}$ is demonstrating how important role that a molecule plays in this independent process of the pathogenesis of non-EAOC. A hub gene is a gene that plays an important role in biological processes. In related pathways, the regulation of other genes is often affected by this gene. In this study, we used a public database to screen for differential genes in ovarian cancer and OE tissues and to identify hub genes among these genes. If there is a common hub gene between $\mathrm{OE}$ and ovarian cancer and if there is a significant difference in the expression of the gene in the above-mentioned tissues, then the gene may play an important role in the malignant transformation of the EC.

According to the screening results of DEGs, the expression of RRM2 was low in the EC and high in the EU and cancer tissues. IHC revealed that RRM2 protein was expressed in the EU, EC, and cancer tissues of EAOC patients, and there was no significant low expression of RRM2 protein in EC. The expression of RRM2 in the EC of EAOC patients was contrary to the results of previous data analysis. That further 
supports our assumption that the expression of RRM2 in the EC with malignant tendency will continue to be upregulated during the malignant transformation of the EC. However, the expression of RRM2 in the EC without malignant tendency will remain at a low level. Furthermore, we compared the EC of patients with EAOC and OE. The results showed that the mRNA expression and IHC score of RRM2 in the EC were significantly higher in the EAOC group than in the OE group. Because the requirement for extracting RNA from FFPE is too high, we only extracted RNA from FFPE tissue sections in the past 5 years, which greatly limited the number of our available RNA samples, resulting in low accuracy in the statistical analysis of mRNA expression frequency. Therefore, it is necessary to quantify the RNA in the tissue samples of additional EAOC patients to confirm that the mRNA expression of RRM2 in the EC is higher in EAOC patients than in OE patients.

RRM2 is related to the cell cycle and mitosis. The downregulation of RRM2 in the EC indicates a low cell proliferation rate, whereas upregulated RRM2 expression in the EC indicates active cell proliferation. Ki-67 is an indicator of cell proliferation. The expression of Ki-67 and RRM2 was examined in the EC of the same patients with EAOC. We found that when the expression of Ki-67 was enhanced, the expression of RRM2 was significant. The overexpression of RRM2 can lead to the active proliferation of histiocytes. Therefore, the high expression of RRM2 in the EC suggests that it may contribute to malignant transformation.

The surgical specimens of patients with OE are usually stained only with $\mathrm{HE}$ and do not require IHC. Patients with OE generally only undergo gynecological color Doppler ultrasound as a postoperative reexamination tool. Unexpected, some patients have been diagnosed with EAOC a few years after surgery for OE, especially among young patients with $\mathrm{OE}$ who choose the surgical method to remove only the EC to preserve the ovaries. However, extremely small lesions in the EC cannot be detected by clinicians with naked the eye, which may be a potential risk of the occurrence of EAOC. The overexpression of RRM2 may play an important role in the malignant transformation of OE. Therefore, IHC for RRM2 should be performed on the postoperative pathological tissues of patients with $\mathrm{OE}$, especially for women who have chosen to undergo ovary-sparing surgery. If the expression of RRM2 is increased, the detection of tumor markers related to ovarian cancer is recommended during re-examination. If economic conditions permit, these patients can also undergo an additional pelvic MRI examination once a year. Therefore, the malignant transformation of the EC can be detected in time, and further progress of the disease can be prevented.

In summary, we screened for the differentially expressed hub gene RRM2 in OE and EAOC using a public database. We examined the expression of RRM2 protein in the cancer tissues, EC, and EU of patients with EAOC (CCOC and EOC). The EC samples of the patients were stained with RRM2 and Ki-67, and the RNA from FFPE tissue sections was extracted. The expression of RRM2 in the EC and cell proliferation of EAOC and OE patients were analyzed. The results showed that the mRNA and protein expression levels of RRM2 were related to the pathological features of the tumor. We believe that the overexpression of RRM2 may be a pathogenic factor leading to replication imbalance and error-prone replication in the dNTP pool, thus promoting genomic instability and subsequent malignant transformation, which could lead to 
abnormal cell proliferation. Therefore, RRM2 may be used as a marker of OE malignant transformation, which contribute to the research of the mechanism of the malignant transformation of OE. Nevertheless, this mechanism requires further investigation.

\section{Abbreviations}

RRM2:ribonucleotide reductase M2;EC:ectopic endometrium;OE:ovarian endometriosis ;GEO:Gene Expression Omnibus;EAOC:endometriosis-associated ovarian cancers ; IHC:immunohistochemistry;RTqPCR:real-time quantitative polymerase chain reaction;EU:eutopic endometrium;EMS:endometriosis;CCOC:clear cell ovarian carcinoma ; EOC:endometrioid ovarian carcinoma;DEGszzzz:differentially expressed genes;PPl:protein-protein interaction;RNR:Ribonucleotide reductase; dNTP:ribonucleoside 5'-diphosphate to 2'-deoxyribonucleoside 5'-triphosphate;OIS:oncogeneinduced senescence ;FFPE:Formalin-fixed, paraffin-embedded

\section{Declarations}

\section{Acknowledgements}

This work was supported by the Tianjin Central Hospital of Gynecology Obsterics.

\section{Author Contributions}

Yuanjing Hu contributed to the study conception and design. Material preparation, data collection and analysis were performed by Binkai Yang. TianWang, NaLi, and Wenwen Zhang contributed to revisions. All authors read and approved the final manuscript.

\section{Fundings}

This work was supported by the Major Science and Technology Projects in Tianjin $\Downarrow$ grant number 17ZXMFSY00160 and National Natural Science Foundation of China, grant number 81802605 .

\section{Ethics approval and consent to participate}

Approval was obtained from the ethics committee of Tianjin Central Hospital of Gynecology Obsterics. The procedures used in this study adhere to the tenets of the Declaration of Helsinki.

\section{Consent for publication}

All authors read the fnal manuscript and agreed to publish it.

\section{Availability of data and materials}

All data are included in the article. 


\section{Conflict of Interest}

The authors declare that the research was conducted in the absence of any commercial or financial relationships that could be construed as a potential conflict of interest.

\section{References}

1. Schmidt CL: Endometriosis: a reappraisal of pathogenesis and treatment. FERTIL STERIL 1985, 44(2):157.

2. Sun Y, Che X, Zhu L, Zhao M, Fu G, Huang X, Xu H, Hu F, Zhang X: Pigment epithelium derived factor inhibits the growth of human endometrial implants in nude mice and of ovarian endometriotic stromal cells in vitro. PLOS ONE 2012, 7(9):e45223.

3. Kobayashi $\mathrm{H}$, Higashiura $\mathrm{Y}$, Shigetomi $\mathrm{H}$, Kajihara $\mathrm{H}$ : Pathogenesis of endometriosis: the role of initial infection and subsequent sterile inflammation (Review). MOL MED REP 2014, 9(1):9-15.

4. Giudice LC: Endometriosis. NEW ENGL J MED 2010, 362(25):2389-2398.

5. Nishida M, Watanabe K, Sato N, Ichikawa Y: Malignant transformation of ovarian endometriosis. Gynecol Obstet Invest 2000, 50 Suppl 1:18-25.

6. Seidman JD: Prognostic importance of hyperplasia and atypia in endometriosis. INT J GYNECOL PATHOL 1996, 15(1):1-9.

7. Kobayashi H, Sumimoto K, Kitanaka T, Yamada Y, Sado T, Sakata M, Yoshida S, Kawaguchi R, Kanayama S, Shigetomi $\mathrm{H}$ et al: Ovarian endometrioma-Risks factors of ovarian cancer development. EUR J OBSTET GYN R B 2008, 138(2):187-193.

8. Mandai M, Yamaguchi K, Matsumura N, Baba T, Konishi I: Ovarian cancer in endometriosis: molecular biology, pathology, and clinical management. INT J CLIN ONCOL 2009, 14(5):383-391.

9. Kurman RJ, Carcangiu ML, Herrington CS, Young RH: WHO Classification of Tumours of Female Reproductive Organs., 4th edn. Lyon, France: IARC; 2014.

10. Kurman RJ, Shih I: The Dualistic Model of Ovarian Carcinogenesis. The American Journal of Pathology 2016, 186(4):733-747.

11. Poole EM, Lin WT, Kvaskoff M, De Vivo I, Terry KL, Missmer SA: Endometriosis and risk of ovarian and endometrial cancers in a large prospective cohort of U.S. nurses. CANCER CAUSE CONTROL 2017, 28(5):437-445.

12. Ness RB: Endometriosis and ovarian cancer: Thoughts on shared pathophysiology. AM J OBSTET GYNECOL 2003, 189(1):280-294.

13. Modesitt SC, Tortolero-Luna G, Robinson JB, Gershenson DM, Wolf JK: Ovarian and extraovarian endometriosis-associated cancer. Obstetrics \& Gynecology 2002, 100(4):788-795.

14. Taniguchi F: New knowledge and insights about the malignant transformation of endometriosis. $J$ OBSTET GYNAECOL RE 2017, 43(7):1093-1100. 
15. Jiang $L$, Zhang $M$, Wang $S$, Han $Y$, Fang $X$ : Common and specific gene signatures among three different endometriosis subtypes. PEERJ 2020, 8:e8730.

16. Shen J, Yu S, Sun X, Yin M, Fei J, Zhou J: Identification of key biomarkers associated with development and prognosis in patients with ovarian carcinoma: evidence from bioinformatic analysis. J OVARIAN RES 2019, 12(1).

17. Yang D, He Y, Wu B, Deng Y, Wang N, Li M, Liu Y: Integrated bioinformatics analysis for the screening of hub genes and therapeutic drugs in ovarian cancer. J OVARIAN RES 2020, 13(1).

18. Nordlund P, Reichard P: Ribonucleotide reductases. ANNU REV BIOCHEM 2006, 75:681-706.

19. Engstrom Y, Eriksson S, Jildevik I, Skog S, Thelander L, Tribukait B: Cell cycle-dependent expression of mammalian ribonucleotide reductase. Differential regulation of the two subunits. J BIOL CHEM 1985, 260(16):9114-9116.

20. Furuta $\mathrm{E}$, Okuda $\mathrm{H}$, Kobayashi $\mathrm{A}$, Watabe K: Metabolic genes in cancer: Their roles in tumor progression and clinical implications. Biochimica et biophysica acta. Reviews on cancer 2010, 1805(2):141-152.

21. Shao J, Zhou B, Chu B, Yen Y: Ribonucleotide reductase inhibitors and future drug design. CURR CANCER DRUG TAR 2006, 6(5):409.

22. Aird KM, Zhang G, Li H, Tu Z, Bitler BG, Garipov A, Wu H, Wei Z, Wagner SN, Herlyn M et al: Suppression of nucleotide metabolism underlies the establishment and maintenance of oncogeneinduced senescence. CELL REP 2013, 3(4):1252-1265.

23. Duxbury MS, Ito $H$, Zinner MJ, Ashley SW, Whang EE: RNA interference targeting the M2 subunit of ribonucleotide reductase enhances pancreatic adenocarcinoma chemosensitivity to gemcitabine. ONCOGENE 2004, 23(8):1539-1548.

24. Itoi T, Sofuni A, Fukushima N, Itokawa F, Tsuchiya T, Kurihara T, Moriyasu F, Tsuchida A, Kasuya K: Ribonucleotide reductase subunit M2 mRNA expression in pretreatment biopsies obtained from unresectable pancreatic carcinomas. J GASTROENTEROL 2007, 42(5):389-394.

25. Duxbury MS, Whang EE: RRM2 induces NF-KB-dependent MMP-9 activation and enhances cellular invasiveness. BIOCHEM BIOPH RES CO 2007, 354(1):190-196.

26. Liu X, Zhou B, Xue L, Yen F, Chu P, Un F, Yen Y: Ribonucleotide reductase subunits M2 and p53R2 are potential biomarkers for metastasis of colon cancer. Clin Colorectal Cancer 2007, 6(5):374-381.

27. Zhang K, Hu S, Wu J, Chen L, Lu J, Wang X, Liu X, Zhou B, Yen Y: Overexpression of RRM2 decreases thrombspondin-1 and increases VEGF production in human cancer cells in vitro and in vivo: implication of RRM2 in angiogenesis. MOL CANCER 2009, 8(1):11.

28. Souglakos J, Boukovinas I, Taron M, Mendez P, Mavroudis D, Tripaki M, Hatzidaki D, Koutsopoulos A, Stathopoulos E, Georgoulias V et al: Ribonucleotide reductase subunits M1 and M2 mRNA expression levels and clinical outcome of lung adenocarcinoma patients treated with docetaxel/gemcitabine. $\mathrm{Br} J$ Cancer 2008, 98(10):1710-1715.

29. Mazzu YZ, Armenia J, Chakraborty G, Yoshikawa Y, Coggins SA, Nandakumar S, Gerke TA, Pomerantz MM, Qiu X, Zhao H et al: A Novel Mechanism Driving Poor-Prognosis Prostate Cancer: 
Overexpression of the DNA Repair Gene, Ribonucleotide Reductase Small Subunit M2 (RRM2). CLIN CANCER RES 2019, 25(14):4480-4492.

30. Aird KM, Li H, Xin F, Konstantinopoulos PA, Zhang R: Identification of ribonucleotide reductase M2 as a potential target for pro-senescence therapy in epithelial ovarian cancer. CELL CYCLE 2013, 13(2):199-207.

31. Aird KM, Zhang G, Li H, Tu Z, Bitler BG, Garipov A, Wu H, Wei Z, Wagner SN, Herlyn M et al: Suppression of Nucleotide Metabolism Underlies the Establishment and Maintenance of OncogeneInduced Senescence. CELL REP 2013, 3(4):1252-1265.

\section{Figures}

a

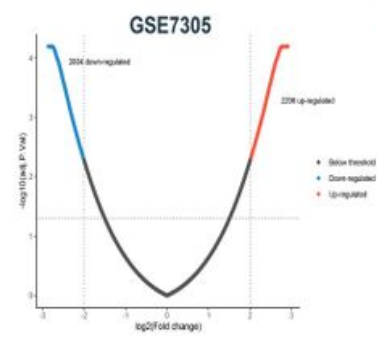

c

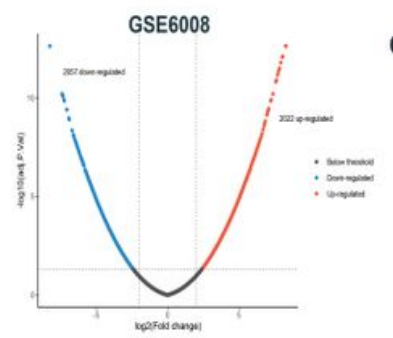

b

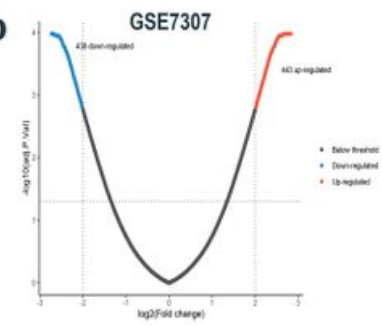

d

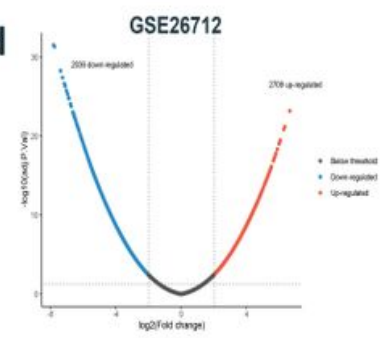

e

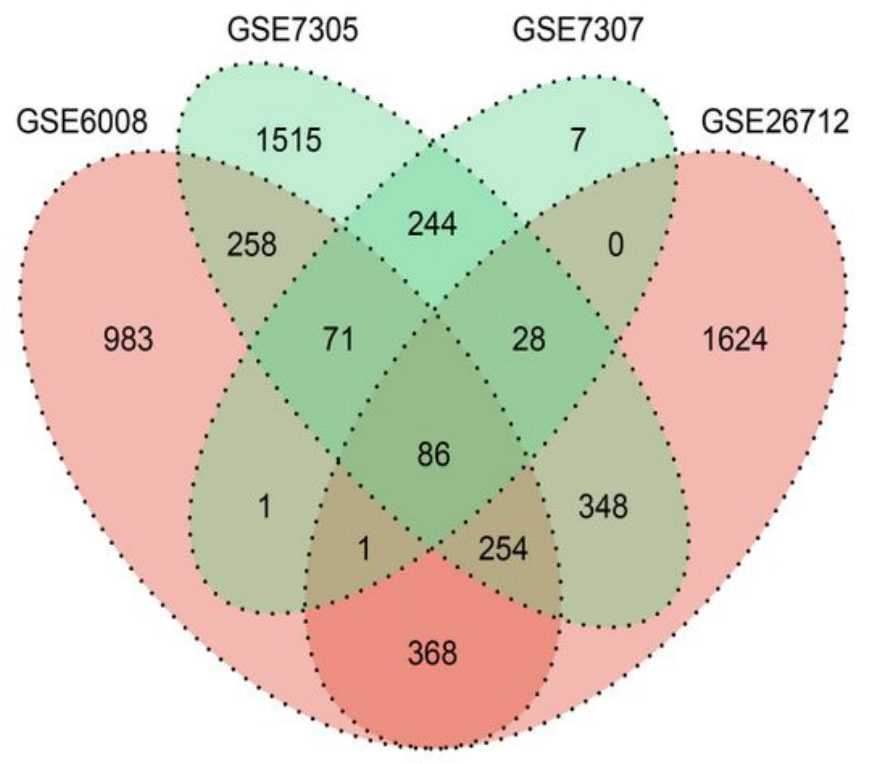

\section{Figure 1}

Identification and screening of DEGs ( $a$ and $b$ ): Volcano plot of DEGs between OE tissues and normal endometrial tissues in each dataset. ( $c$ and d): Volcano plot of DEGs between ovarian cancer tissues and normal ovarian tissues in each dataset. $\mathrm{P}<0.05$ and $|\log \mathrm{FC}|>2$ were considered as statistically significant. (e): Venn diagram for overlapping DEGs from selected datasets. 
a

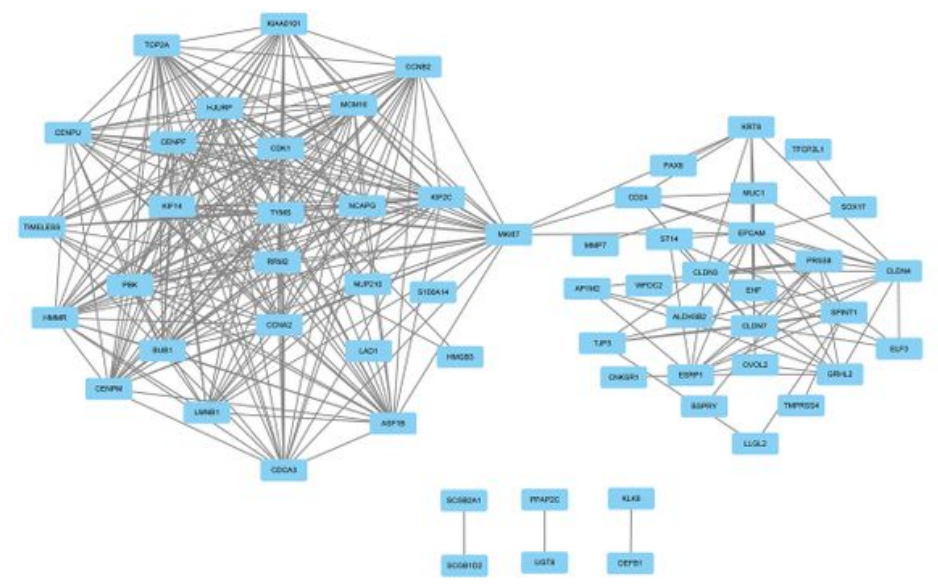

c

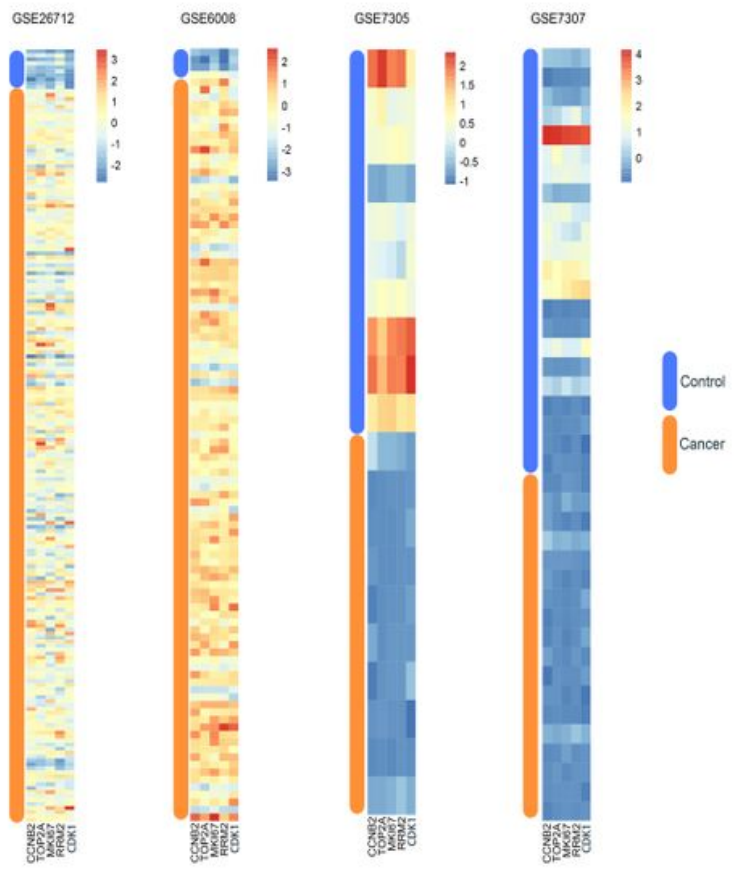

b

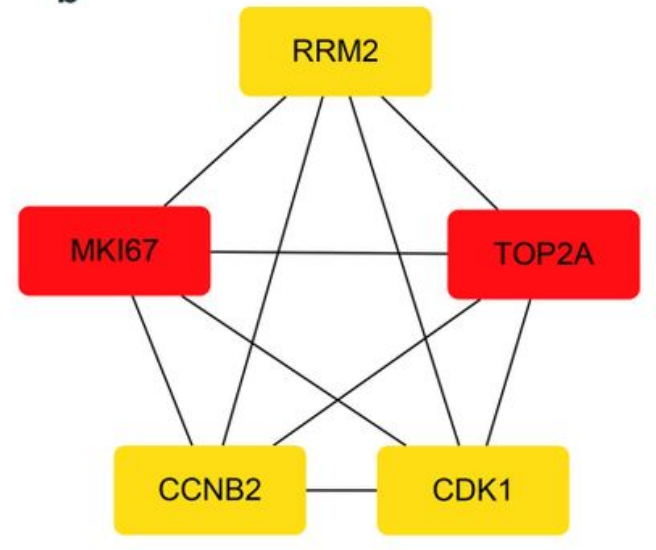

\section{Figure 2}

PPI network and identification of hub nodes (a): A total of 86 DEGs were visualized in the DEGs PPI network complex: the nodes represent proteins, and the edges represent the interaction of the proteins. (b):

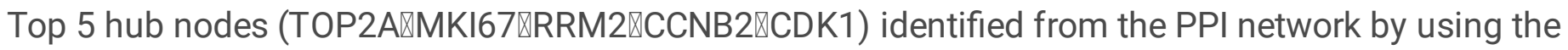
Degree method in plugin Cytohubba. The nodes color changes gradually from yellow to red in ascending

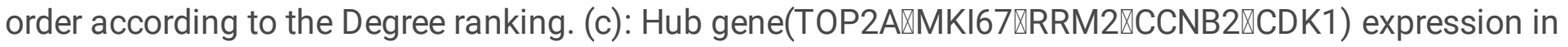
the GSE6008, GSE26712, GSE7305 and GSE7307. 


\section{RRM2}

\section{eutopic endometrial}
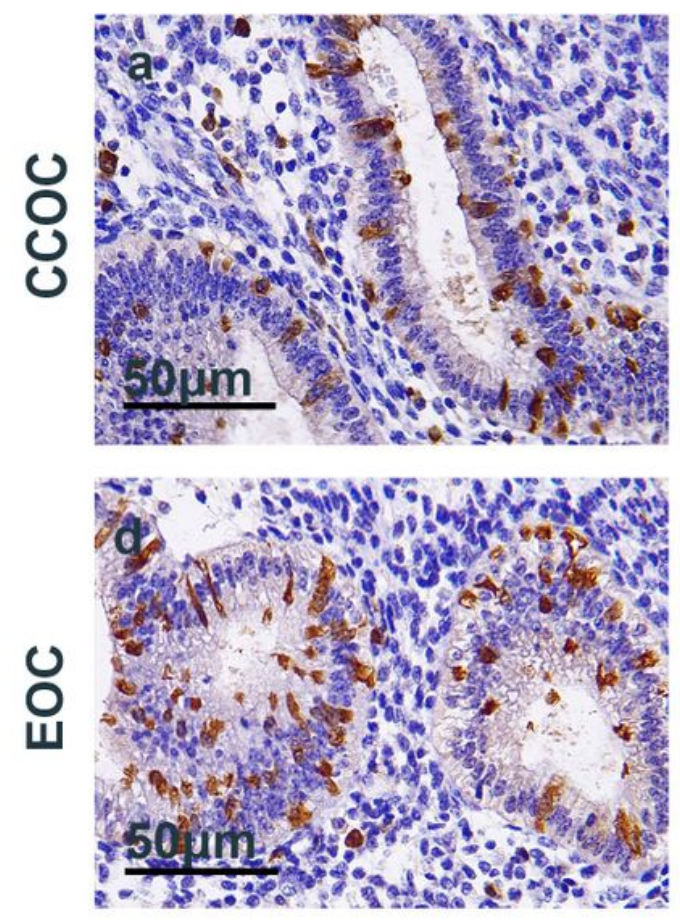

ectopic endometrial
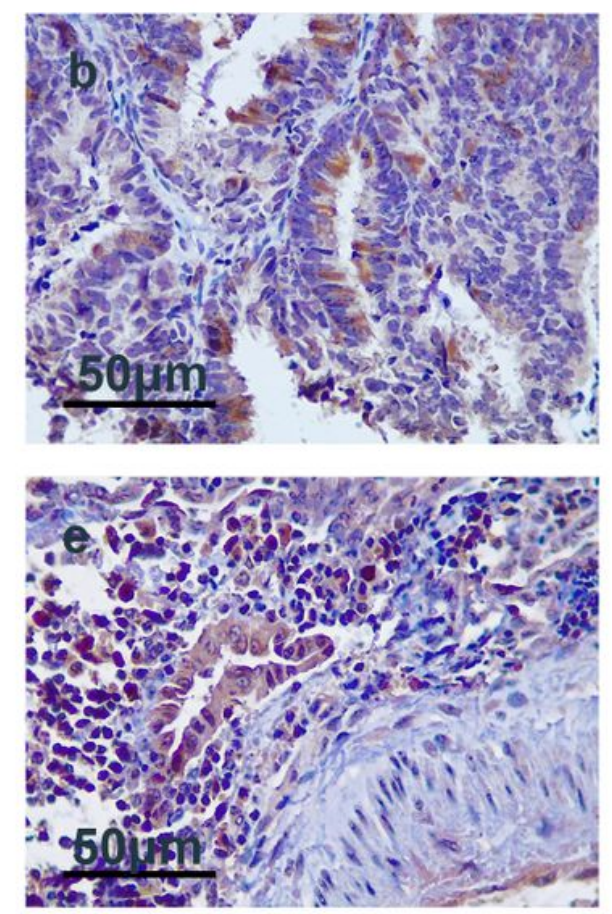

\section{carcinoma}
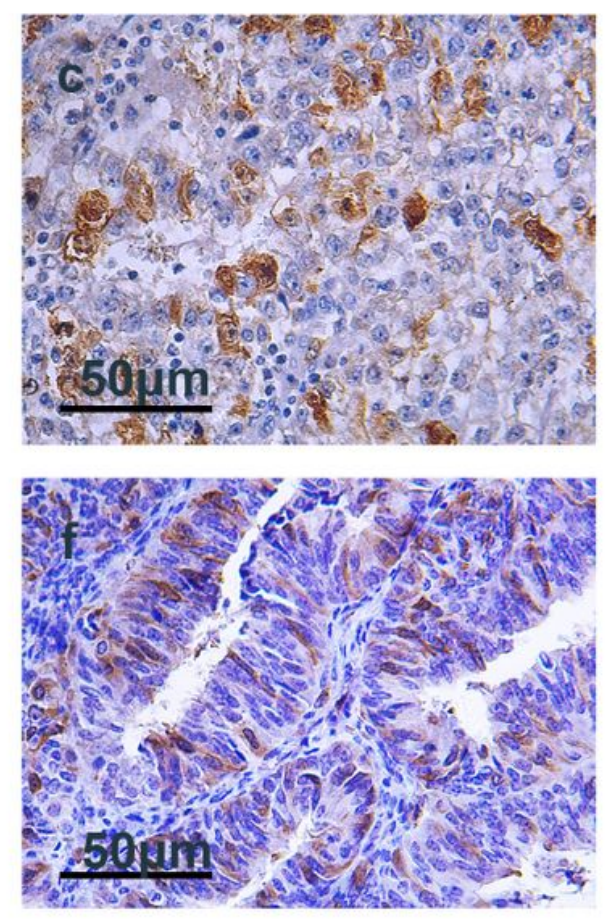

Figure 3

The expression of RRM2 in EU, EC,and EAOC cancer tissues from patient with $\mathrm{CCOC}$ and patient with EOC (a): EU of patients with CCOC. (b): EC of patients with CCOC. (c): Cancerous tissues of patients with CCOC. (d): EU of patients with EOC. (e): EC of patients with EOC. (f): Cancerous tissues of patients with EOC. (original magnification $\times 400)$. Scalebars $50 \mu \mathrm{m}$ in $(a-f)$ 
a

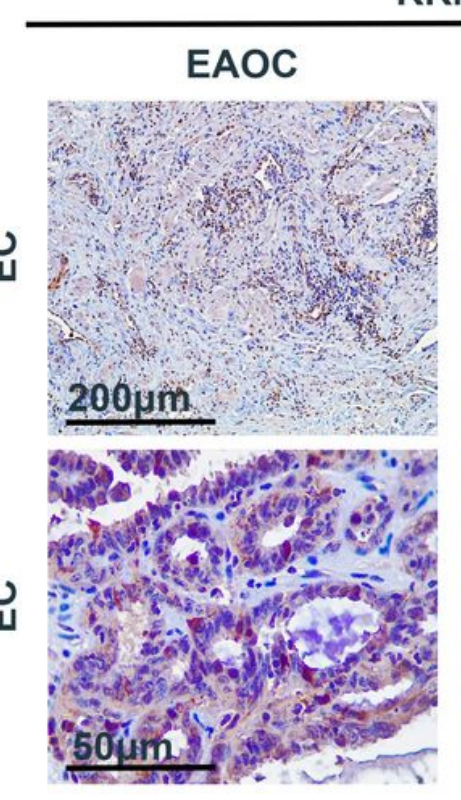

b

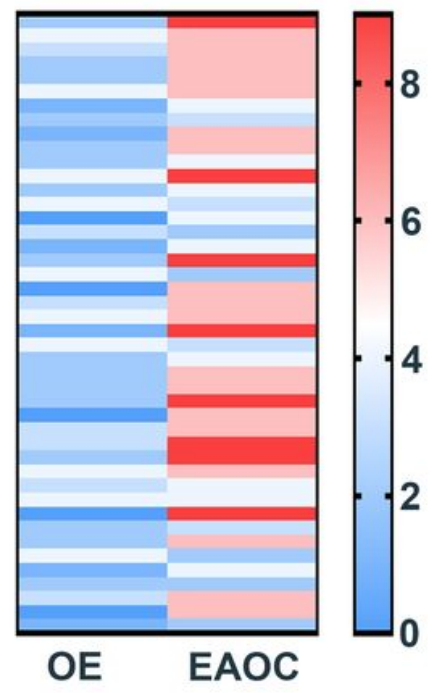

$\mathrm{N}=44 \quad \mathrm{p}<0.01$

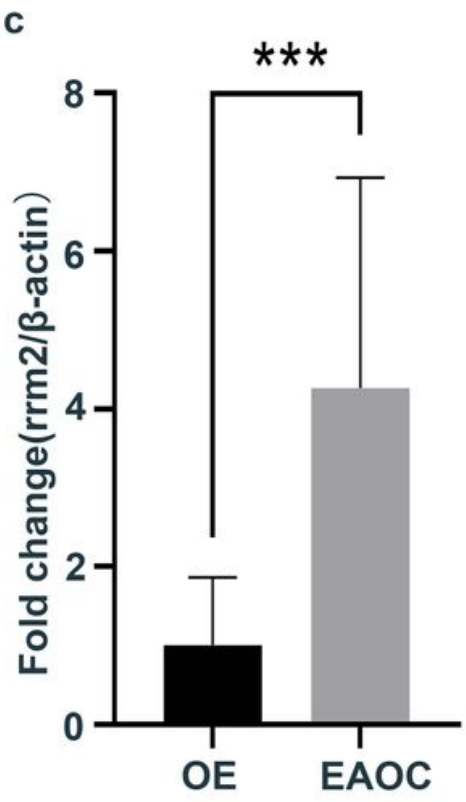

Figure 4

Expression of RRM2 in EC of EAOC and OE (a): IHC of RRM2 in EC from EAOC and OE patients. (b): The immunohistochemical score of RRM2 in EC of 44 patients with EAOC and 44 patients with OE. Blue is low expression, white is medium expression, and red is high expression. (c): Detection of mRNA level of RRM2 in EC of 15 cases of EAOC and 15 cases of OE by RT-qPCR. $\star \star \star$ indicates $P<0.001$

\section{EAOC}

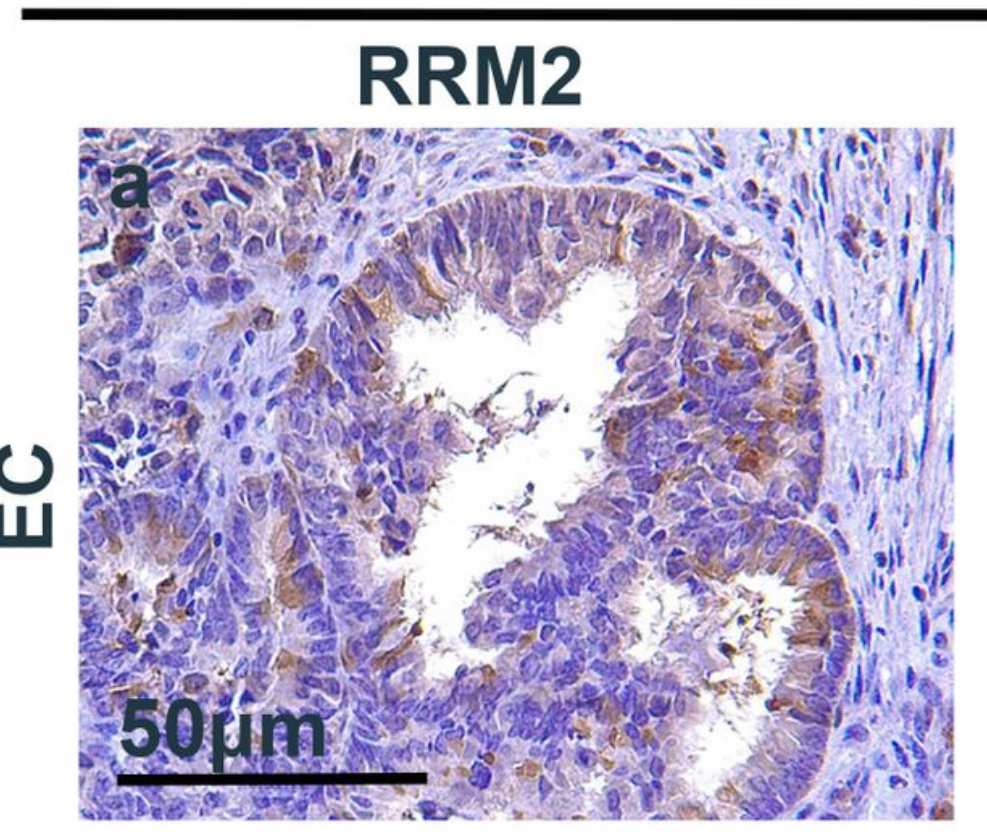

\section{Ki-67}

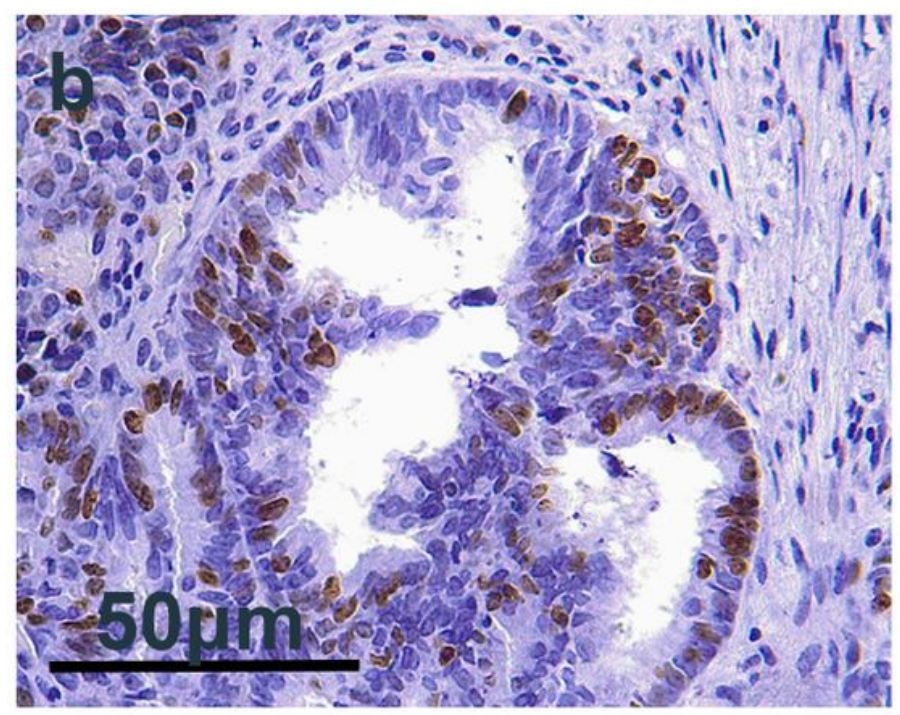


Figure 5

Expression of RRM2 and $\mathrm{Ki}-67$ in EC of EAOC from an EAOC patient (a): Expression of RRM2 in EC patients with EAOC. (b): Expression of Ki-67 in EC patients with EAOC. (original magnification $\times 400$ ). Scalebars $50 \mu \mathrm{m}$ in (a and $b)$ 